\title{
Prediction using learning coefficients for efficient operation of deregulated electricity market
}

\author{
Perumal Nallagownden ${ }^{1}$, Syafrudin Masri ${ }^{2}$ \\ ${ }^{1}$ Electrical \& Electronic Engineering Dept, University Technology Petronas, Malaysia. \\ perumal@petronas.com.my \\ ²Electrical Engineering Dept, University Sains Malaysia. syaf@org.usm.my
}

\begin{abstract}
Deregulation and restructuring of electric power industry is occurring in many parts of the world. It is aimed at introducing competition in the supply and retail side of the industry, while maintaining control over the transmission lines. Tracing methodology hence had been introduced to overcome problems related to the Marginal pricing of transmission costs. This paper first analyses of the proportional method which leads to a redefined power tracing method, and then further refines the proposed prediction method by establishing trends of the learning coefficients, using them to examine the relationship between accuracy and number of samples taken. Response of individual generators to change in demand and the corresponding associated losses are also presented. MATLAB with matpower4.0b extension was used to present the study on the IEEE 24bus RTS.
\end{abstract}

Keywords: Prediction, Proportional Tracing Methodology, Sending Algorithm, Receiving Algorithm, Regression

\section{INTRODUCTION}

DEREGULATION of the electric power industry was aimed at creating competition and improving efficiency of the electric power industry. The reform has allowed entry of multiple players in their respective functions: power producers, retailers, TSOs, instead of the traditional monopoly by a single utility [2], thereby giving customers a choice of purchasing energy from the supplier of their choice without impacting reliability.

In the past, it has been assumed that electricity and its delivery has been intermingled and cannot be unbundled. Electricity has been viewed as a product used only at the point of delivery. A closer analysis of an energy system is seen to be a combination of several functional building blocks: primarily generation, transmission and retailing. This has a big impact on the industry as it requires not only a host of ownership, organization and functional changes, but also a change in power industry management. Contrary to traditional vertically integrated system, monopoly is fully intended to be removed from generation, transmission and distribution. As a result generation and distribution are competitive, with many different companies vying for the business. Their price and practices are not regulated any more.
A deregulated energy market model is governed by the law of demand and supply. Of course the proposition is not for a total free market, especially in its inception stage but with the existence of a regulating body, known as an ISO [3], who plays the role of a supervisor for system operation, planning and security It has operational control authority over the whole power system and normally operates and maintains the transmission lines. Deregulation not only reforms the way business transaction is performed in the energy market, but also the gencos' business strategy by only supplying sufficient power to meet the demand at a given point of time to prevent unrecovered generation cost [4]. No commodity can be traded however, unless there are appropriate arrangements for its delivery and pricing. Transmission pricing plays a crucial role in determining whether providing transmission services is economically beneficail to all parties involved. However, all cost saving measures has to account for system reliability because in any event of supply shortage, a possible major black out may be triggered

In a fully deregulated energy market the ISO's are required to publish the hourly load forcasts and actual loads. To facilitate market transpapercy the ISO operators must calculate hourly requirements 24 hours in advance and post the information for the 
market. The ISO will facilitate a mechanism that allows a process of bidding to take place. Since a purchase bid need to be prepared by a retailer for a power demend to come up at a later time of the day, based on current network flow distribution, it is required to predict generations participating in meeting a retailer's future demend, predict capacity utilization of alternate bulk transmission paths supplying this demand.

Prediction hence plays an even more crucial role in a deregulated market since no genco will be willing to generate power in excess than what is demanded. The question now is how to harness the information provided by any of those afore mentioned tracing methods in performing efficient forecasting in a deregulated energy market.

This paper presents an alternative form of the Proportional Tracing Methodology, which uses the power flow of the power system under study to model the matrices, and the further establishment of the learning coefficients as a valid prediction method by performing trends analysis. On the sideline, the analysis of the trends was found to contain information which could aid retailers or gencos in performing an aided economic decision.

\section{Proportional Tracing Methodology}

In contrary to common believe that electric power cannot be traced in a meshed network apart from the total current input and total current output at a node according to Kirchhoff's Current Law, J. Bialek [5] proved that the proportional method could be used to assess quantity of power wheeled from a particular generator to a particular retailer in proportion to the demanded power at retailer end, which simultaneously assesses the contribution of individual generators or loads to individual line flows and line losses. Realisation of this method is done through two algorithms: the Upstream Algorithm which traces the gross flow (inflow) and the Downstream Algorithm which traces the net flow (outflow) [5].

\section{Upstream Algorithm}

$\mathrm{P}_{i}$, the inflow to the $i^{\text {th }}$ bus, is the sum of all the inflows through the lines connected to the bus and the local bus injection

$$
\mathrm{P}_{i}=\left(\sum_{j \in \eta}\left|\mathrm{P}_{i-j}\right|\right)+\mathrm{P}_{G i} \quad \text { for } \mathrm{i}=1,2, \ldots \mathrm{n}
$$

where $\eta$ is the set of nodes directly supplying node $i$, implying power flow towards $i^{\text {th }}$ node. If the line losses are neglected, then $\left|P_{j-i}\right|=\left|P_{i-j}\right|$. Equation (1) can be further expanded to become:

$$
\mathrm{P}_{\mathrm{i}}=\left(\sum_{j \in \eta}\left|\frac{P_{j-i}}{P_{j}} P_{j}\right|\right)+\mathrm{P}_{G i} \quad \text { for } \mathrm{i}=1,2, \ldots \mathrm{n}
$$

By defining $c_{j i}=\left|P_{j-i}\right| P_{j}$ to express relationship between line flow and the nodal flow at the $j^{\text {th }}$ node, using proportional sharing principle $\left|P_{j-i}\right|=c_{j i} P_{j}$, substituting this in (2) yields:

$$
\left(\mathrm{P}_{i}-\sum_{j \in \eta} c_{j i} \mathrm{P}_{j}\right)=\mathrm{P}_{G i} \text { or } \mathrm{A}_{u} \mathrm{P}=\mathrm{P}_{G}
$$

$P$ is the vector of gross nodal flows; $P_{G}$ is the vector of nodal generations, while $A_{u}$ is called the Upstream Matrix, which elements can be generalized as follow:

$$
\left[\mathrm{A}_{u}\right]_{i j}= \begin{cases}1 & \text { for } i=j \\ -c_{j i}=-\frac{\left|\mathrm{P}_{j-i}\right|}{\mathrm{P}_{j}} & \text { for } j \in \eta \\ 0 & \text { otherwise }\end{cases}
$$

The $i^{\text {th }}$ element of $\mathrm{P}=\mathrm{A}_{u}^{-1} \mathrm{P}_{G}$ shows the participation of the $k^{\text {th }}$ generation to the $i^{\text {th }}$ nodal flow and determines the relative participation of the nodal generations in meeting a retailer's demand, given by:

$$
\mathrm{P}_{i}=\sum_{k=1}^{n}\left[\mathrm{~A}_{\mathrm{u}}^{-1}\right]_{i k} \mathrm{P}_{G k} \quad \text { for } i=1,2, \ldots . n
$$

Finally, load demand at the $i^{\text {th }}$ bus, applying the proportional methodology is given by:

$$
\begin{aligned}
& \mathrm{P}_{L i}=\frac{\mathrm{P}_{L i}}{\mathrm{P}_{i}} \mathrm{P}_{i} \text { or } \\
& \mathrm{P}_{L i}=\left(\frac{\mathrm{P}_{L i}}{\mathrm{P}_{i}} \sum_{\mathrm{k}=1}^{\mathrm{n}}\left[\mathrm{A}_{\mathrm{u}}^{-1}\right]_{i j}\right) \mathrm{P}_{G k} \text { from } i=1,2, \ldots n
\end{aligned}
$$

Equation (6) shows the gross demand at node $i$. Share of the output of the $i^{\text {th }}$ generator used to supply the $k^{\text {th }}$ load demand at the point of generation is indicated by the respective $k-i$ element of the $A_{u}^{-1}$ matrix. It can be used to trace where the power of a generator goes to.

\section{Downstream Algorithm}

$\mathrm{P}_{i}$, the outflow to the $i^{\text {th }}$ bus, is the sum of all the outflows through the lines connected to the bus and the local bus load

$$
\mathrm{P}_{\mathrm{i}}=\left(\sum_{l \in \mu}\left|\mathrm{P}_{\mathrm{i}-\ell}\right|\right)+\mathrm{P}_{\mathrm{Li}} \quad \text { for } \mathrm{i}=1,2, \ldots \mathrm{n}
$$

where $\mu$ is the set of nodes directly supplied from node $i$, implying power flowing from the $i^{\text {th }}$ node. If the 
line losses are neglected, then $\left|\mathrm{P}_{l-i}\right|=\left|\mathrm{P}_{i-1}\right|$. Equation (7) can be further expanded into:

$$
\mathrm{P}_{\mathrm{i}}=\left(\sum_{l \in \mu}\left|\frac{P_{l-i}}{P_{l_{l}}} P_{l}\right|\right)+\mathrm{P}_{\mathrm{Li}} \quad \text { for } \mathrm{i}=1,2 \ldots ., \mathrm{n}
$$

Defining $c_{l i}=\left|P_{l-i}\right| / P_{l}$ expressing relationship between line flow and the nodal flow at the $I^{\text {th }}$ node and using proportional sharing principle, $\left|P_{l-i}\right|=c_{l i} P_{l}$. Substituting this in (8) yields

$$
\left(\mathrm{P}_{i}-\sum_{l \in \mu} C_{l i} \mathrm{P}_{\ell}\right)=\mathrm{P}_{L i} \quad \text { or } \mathrm{A}_{d} \mathrm{P}=\mathrm{P}_{L}
$$

$P$ is the vector of net nodal powers; $P_{L}$ is the vector of nodal load demands, while $A_{d}$ is called the Downstream Matrix, which elements can be generalized as follow:

$$
\left[\mathrm{A}_{d}\right]_{i \ell}= \begin{cases}1 & \text { for } i=j \\ -c_{\ell i}=-\frac{\left|\mathrm{P}_{\ell-i}\right|}{\mathrm{P}_{\ell}} & \text { for } j \in \mu \\ 0 & \text { otherwise }\end{cases}
$$

The $i^{\text {th }}$ element of $\mathrm{P}=\mathrm{A}_{d}{ }^{-1} \mathrm{P}_{L}$ shows the distribution of the $i^{\text {th }}$ nodal power between all the loads in the system. In summation form,

$$
\mathrm{P}_{i}=\sum_{k=1}^{n}\left[\mathrm{~A}_{\mathrm{d}}^{-1}\right]_{i k} \mathrm{P}_{\mathrm{Lk}} \quad \text { for } i=1,2, \ldots . . n
$$

Finally, nodal generation as an inflow at the $i^{\text {th }}$ bus, applying the proportional methodology is given by:

$$
\begin{aligned}
& \mathrm{P}_{G i}=\frac{\mathrm{P}_{G i}}{\mathrm{P}_{i}} \mathrm{P}_{i} \text { or } \\
& \mathrm{P}_{G i}=\left(\frac{\mathrm{P}_{G i}}{\mathrm{P}_{i}} \sum_{\mathrm{k}=1}^{\mathrm{n}}\left[\mathrm{A}_{\mathrm{d}}^{-1}\right]_{i j}\right) \mathrm{P}_{L k} \text { from } i=1,2, \ldots n
\end{aligned}
$$

Equation (12) shows the net generation at node $i$. Contribution of the $k^{\text {th }}$ generator to the $i^{\text {th }}$ load demand at the point of consumption is indicated by the respective $i-k$ element of the $A_{d}^{-1}$ matrix. It can be used to trace where the power of a specific load comes from.

\section{The Sending And Receiving Algorithm}

Main issue with Proportional tracing is the lack of physical sense to the mathematics proposed in the Upstream and Downstream algorithms. It would be desired to be able to implement the proportional method in a more direct manner. The benefit of having a method which resembles the condition of the power system would be ease of results interpretation. This is in specific reference to both the Upstream and Downstream matrices which do not posses this quality. The ability to directly relate the matrix elements to the matrices would allow direct interpretation of data. Also consistency in the representation of rows and columns of those matrices would eliminate confusions or possible errors.

\section{The Sending Algorithm}

The diagonal elements of the $A_{u}$ and the $A_{d}$ matrices are always one because it is essentially the bus power level of bus $i$ divided itself.

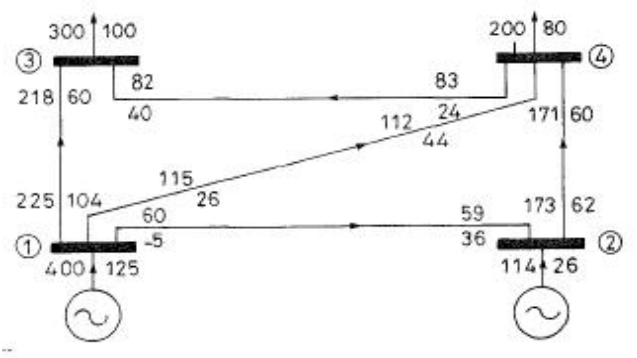

Fig. 1. 4-bus test system, from [5]

In reference to the 4-bus system used in [5] depicted in Figure 1, this condition is illustrated in matrix form,

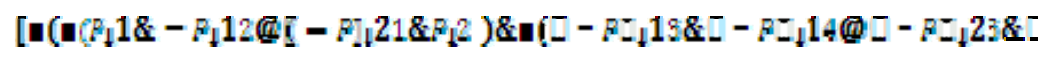

Defining the first matrix as the sending matrix, $A_{s}$ where the column represents the sending bus while row represents the receiving bus. This matrix describes how much power bus $j$ is contributing to bus $i$. The second matrix on the LHS is called the inverse nodal through flow power, which is sum of either the inflow of the outflow of bus $i$. Demonstrating on the test system,

$\left[\begin{array}{cccc}400 & 0 & 0 & 0 \\ -60 & 173 & 0 & 0 \\ -225 & 0 & 900 & -83 \\ -115 & -173 & 0 & 283\end{array}\right]\left[\begin{array}{cccc}400 & 0 & 0 & 0 \\ 0 & 173 & 0 & 0 \\ 0 & 0 & 900 & 0 \\ 0 & 0 & 0 & 283\end{array}\right]^{-1}\left[\begin{array}{l}F_{1} \\ F_{2} \\ F_{2} \\ F_{4}\end{array}\right]=\left[\begin{array}{c}400 \\ 114 \\ 0 \\ 0\end{array}\right]$

Solving the above yields

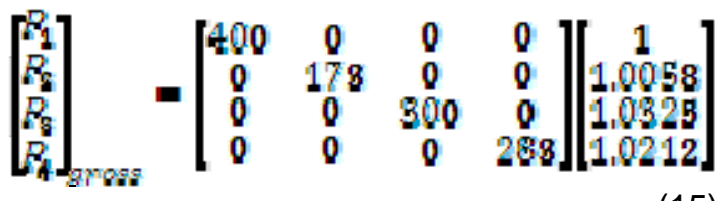

The rightmost matrix is called the loss factor matrix 
(due to wheeling), where element $(k, 1)$ corresponds to losses associated with supplying load $k$, where $L_{g}:(1.0325-1) \times 300=9.75 M W$ and
Breaking down the losses with respect to transactions between generator $k$ and load $i$ : $L_{4}:(1,0212-1) \times 200=4: 24, M W$.

TABLE I

BREAKDOWN OF GENERATOR'S CONTRIBUTION TO LOAD'S DEMAND

\begin{tabular}{|l|l|l|l|l|}
\hline & $\mathrm{G} 1$ & $\mathrm{G} 2$ & Total & Loss \\
\hline L3 & $\begin{array}{l}\mathrm{A}_{\mathrm{S}}^{-1}(3,1) \times \mathrm{P}_{\mathrm{L} 3} \times \mathrm{P}_{\mathrm{G} 1} \\
=276.32\end{array}$ & $\begin{array}{l}\mathrm{A}_{\mathrm{s}}^{-1}(3,2) \times \mathrm{P}_{\mathrm{L} 3} \times \mathrm{P}_{\mathrm{G} 2} \\
=33.43\end{array}$ & 309.75 & 9.75 \\
\hline L4 & $\begin{array}{l}\mathrm{A}_{\mathrm{s}}^{-1}(4,1) \times \mathrm{P}_{\mathrm{L3}} \times \mathrm{P}_{\mathrm{G} 1} \\
=123.68\end{array}$ & $\begin{array}{l}\mathrm{As}_{\mathrm{s}}^{-1}(4,2) \times \mathrm{P}_{\mathrm{L} 3} \times \mathrm{P}_{\mathrm{G} 2} \\
=80.56\end{array}$ & 204.24 & 4.24 \\
\hline Total & $400 \mathrm{MW}$ & $114 \mathrm{MW}$ & $514 \mathrm{MW}$ & $14 \mathrm{MW}$ \\
\hline
\end{tabular}

similar manner as in the Sending Algorithm, by

The whole algorithm to some point has been made more intuitive with a direct relation to the nature of a power system, i.e. the matrix was formed by inspecting the power flow. Also, observe in the above table that the rows and columns corresponds to a systematic coordinate system, where the calculation of loss requires the $(x, y)$ element of the $A_{s}{ }^{-1}$ matrix multiplied by the power of generator and load in interest.

\section{The Receiving Algorithm}

The same could be done to the Downstream Algorithm too, but instead of $A_{d}$, a receiving matrix, $A_{r}$ is formed. Using the similar test system,

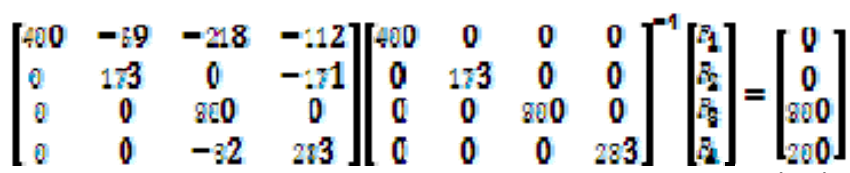

$A_{r}$ takes the transpose form of the $A_{s}$ matrix, but is derived from the power received by bus $j$ from bus $i$. Upon solving,

$$
\left[\begin{array}{l}
R_{1} \\
R_{2} \\
R_{2} \\
R_{1}
\end{array}\right]_{\text {net }}=\left[\begin{array}{cccc}
400 & 0 & 0 & 0 \\
0 & 173 & 0 & 0 \\
0 & 0 & 300 & 0 \\
0 & 0 & 0 & 283
\end{array}\right]\left[\begin{array}{c}
0.9673 \\
0.9849 \\
1 \\
0.9965
\end{array}\right]
$$

The rightmost matrix is called the generator loss factor matrix, where element $(k, 1)$ corresponds to losses associated with generator $k$ supplying all loads,

$\mathrm{G}_{1} 1 \mathrm{1}-0.96939 \times 400=12.28 \mathrm{MW} \quad$ and $\mathrm{G}_{2} \mathrm{I}(1-0,9849) \times 114=1.71 \mathrm{MW}$.

Breakdown of losses with respect to transaction between generator $k$ and load $i$ can be done in the transposing the generator index as rows while load index as column in the table, and using the same referencing in the $A_{r}^{-1}$ matrix.

\section{A. Transaction based loss apportioning}

Since we are more interested in looking at loss incurred by a generator supplying a particular load (generator's (POV) point of view), a sub algorithm is proposed utilizing the two sending and receiving matrices:

$$
A_{S}^{-2}-\left(A_{p^{-1}}^{-2}\right)^{2}=A_{L G S}
$$

where $A_{L(G)}$ is the loss matrix from the generators' POV. $A_{r}^{-1}$ is transposed to transform the shape of the matrix to mimic that of $A_{s}^{-1}$ where the two matrices now carry a common convention. $A_{L(G)}$ always a positive matrix.

\section{TABLE II}

LOSS APPORTIONING TO RESPECTIVE GENERATORS AND LOADS

\begin{tabular}{|c|l|l|c|}
\hline & \multicolumn{1}{|c|}{ G1 } & \multicolumn{1}{c|}{ G2 } & Total \\
\hline L3 & $\mathrm{A}_{\mathrm{L}(\mathrm{G})}{ }^{-1}(3,1) \times \mathrm{P}_{\mathrm{L} 3} \times$ & $\mathrm{A}_{\mathrm{L}(\mathrm{G})}{ }^{-1}(3,2) \times \mathrm{P}_{\mathrm{L} 3} \times$ & 9.76 \\
& $\mathrm{P}_{\mathrm{G} 1}$ & $\mathrm{P}_{\mathrm{G} 2}$ & \\
& $=8.975$ & $=0.7847$ & \\
\hline $\mathrm{L} 4$ & $\mathrm{~A}_{\mathrm{L}(\mathrm{G})}{ }^{-1}(4,1) \times \mathrm{P}_{\mathrm{L} 4} \times$ & $\mathrm{A}_{\mathrm{L}(\mathrm{G})}{ }^{-1}(4,2) \times \mathrm{P}_{\mathrm{L} 4} \times$ & 4.24 \\
& $\mathrm{P}_{\mathrm{G} 1}$ & $\mathrm{P}_{\mathrm{G} 2}$ & \\
& $=3.31$ & $=0.93$ & 14 \\
\hline Total & \multicolumn{1}{|c|}{12.28} & \multicolumn{1}{|c|}{1.71} & 14 \\
\hline
\end{tabular}

Notice the table takes the same form as that of the sending algorithm with the same reference convention (row and column of table to the row and column of matrix).

\section{Prediction Using Learning Coefficient By REgRESSION METHOD}

The usage of learning coefficients in determining generators' optimal dispatch has been established in 
[1], by approximating the heat-rate curve of generator, shown below in Figure 2 with a generalized quadratic relationship in the form of $H\left(P_{G}\right)=\alpha / P_{G}+\beta+\gamma P_{G}[6]$, where $\alpha, \beta$ and $\gamma$ will be solved for respectively by simultaneous equation.

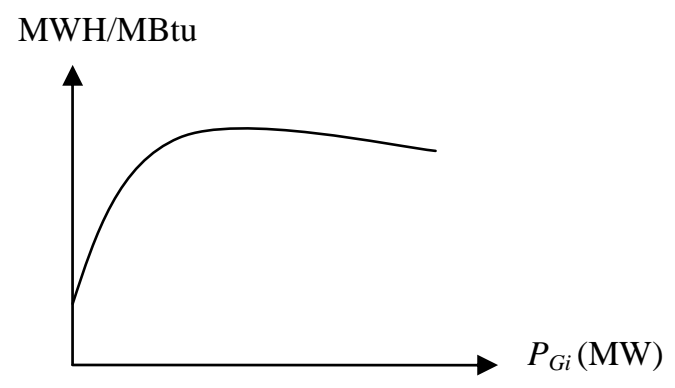

Fig. 2. Generator Heat Rate Curve [6]

The postulation to qualify the evocation of this method is the direct relationship of all the mentioned quantities to the power generated and cost (of generation). Essentially, the governing factor of price per unit of energy is the fuel consumption of the generator in supplying the demanded power, which is governed by the heat rate curve. Transmission losses is tacit within generated power for the reason that power supplied has to meet the demand of retailers, inclusive of losses. It is also straight forward that the greater the power demanded, the greater the generation level will be and subsequently the losses too, up to an allowable limit when no extra generation can take place, as observed from the heat rate curve.

This is extended to the prediction of the (i) share of generations meeting a retailer's demand, and (ii) power loss in a transaction meeting a retailer's demand. Both the relationships assume the form of the aforementioned heat-rate curve of a generator.

Two types of learning coefficients $\left(\alpha_{1}, \beta_{1}, V_{1}\right)$ and $\left(\alpha_{2}, \beta_{2}, \gamma_{2}\right)$, need to be generated at each demand node of the network. Thus, there will be $(n g \times n p g)$ sets of $\left(\alpha_{1}, \beta_{1}, \gamma_{1}\right)$ and $(n g \times n p g)$ sets of $\left(\alpha_{2}, \beta_{2}, \gamma_{2}\right)$ to be generated using real time operating scenarios, during learning exercise. ' $n g$ ' represents number of generations and ' $n p q$ ' represents number of retail or demand points .

Learning relationship between a generator's contribution to a retailers demand at the receiving end

$$
\alpha_{1} / P_{d}+\beta_{1}+\gamma_{1} P_{d}=P_{g d}
$$

Where $P_{d}$ is the total demand at a retailer's point of receipt in per unit (p.u.), $P_{g d}$ is a generator's contribution to a retailers demand at the point of receipt in p.u.

Learning relationship between a retailer's demand and the associated loss in a transaction

$$
\alpha_{2} / P_{d}+\beta_{2}+\gamma_{2} P_{d}=\text { Loss }_{t}
$$

Where $P_{d}$ is the total demand at a retailer's point of

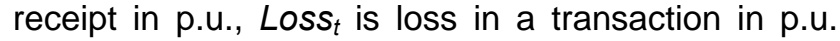
(The difference between a generation's contribution to a demand at the generation end and a generation's contribution to a demand at the load bus, which is the result of the Sending Algorithm less the result of Receiving Algorithm for the same generator and load).

\section{Generation of the coefficients}

Three unknowns require a minimum of three samples for the generation of one set of learning coefficients. The four equations presented above can then be solved respectively, in matrix form:

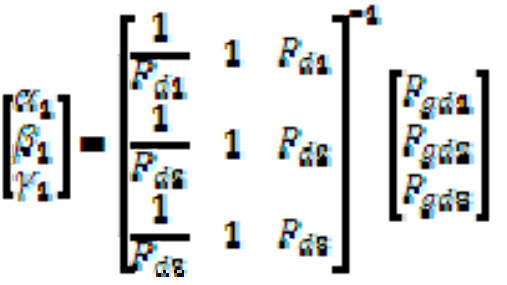

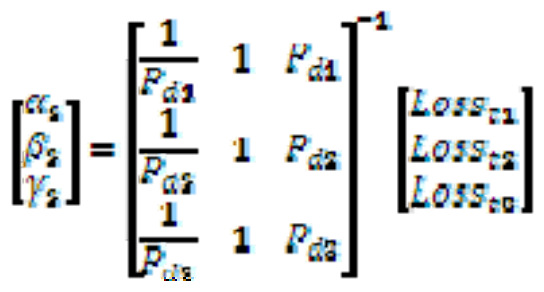

For improved credibility in the learning coefficients, a higher number of samples are to be used. As the number of samples increases, the learning coefficients will be determined using the regression method, where:

$$
x=\left(A^{\top} A x^{-1} A^{\top} b\right.
$$

For matrices or the form $A x=b$. Addition of samples will elicit a row addition to the $A$ and $b$ matrices, changing the dimensions to a rectangular matrix.

\section{TREnd ANALYSis of LeARNing Coefficients}

The IEEE 24 bus Reliability Test System (RTS) [7] was used for this study. Load at bus 8 was chosen as 
Am. J. Sci. Ind. Res., 2010, 1(3): 463-471

load under inspection for prediction to be done, primarily for the reason of its remote location from most of the generators in the IEEE 24 bus RTS. Learning coefficients generation is to be performed specific to the day of the week in a particular season. Monday of the winter weeks: week 52 and 8 is selected at random. Subsequently, five samples are selected by choosing five random hours within the day of week 52 , with the same done for the Monday in week 8.

The five samples of the day are to be handpicked such that they reflect diverse load demands within a day, hence as a rule of thumb, no two consecutive hours are to be selected. Alternatively, the hours can be handpicked by emulating the five various time of day: midnight, beginning of office hour, midday, office dismissal hour and evening for a fair day/night hours representation.

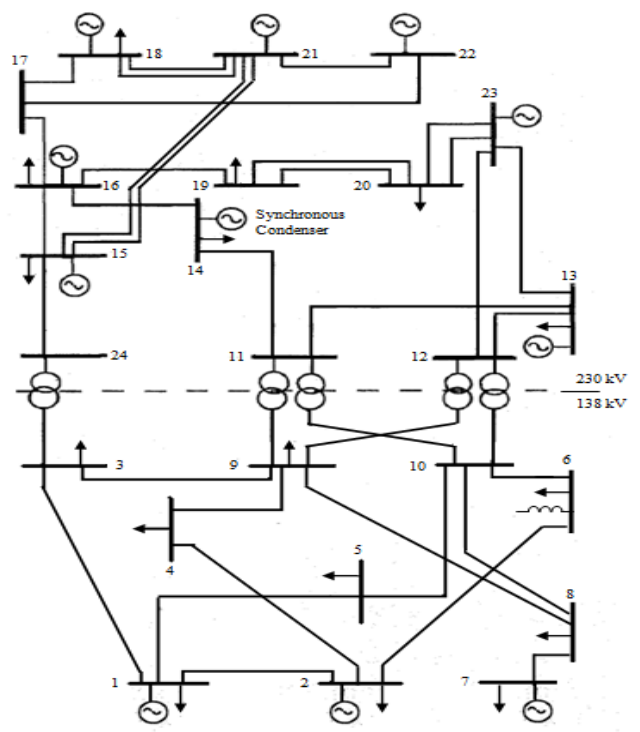

Fig. 3. IEEE 24 bus Reliability Test System [7]

Such procedure is to ensure robustness of the learning coefficients since they are greatly influenced by the spread of the samples picked. At time of simulation, ten samples were chosen from week 8 and 52 as mentioned. Load flow using NewtonRaphson method, followed by the Sending and Receiving Algorithm analysis were performed on each sample respectively, yielding ten sets of such following data, at various $P_{d}$.
TABLE III

TRACING RESULTS FOR $P_{D}=158.2094 M W$

\begin{tabular}{|c|c|c|c|}
\hline \multicolumn{2}{|c|}{ Load: $\underline{\mathbf{8}}$} & \multicolumn{2}{|c|}{ Load Demand 158.2094MW } \\
\hline Gen & Gen End & Retail End & Loss \\
\hline 1 & 0.0000 & 0.0000 & 0.0000 \\
\hline 2 & 0.0000 & 0.0000 & 0.0000 \\
\hline 7 & 115.0000 & 112.9032 & 2.0968 \\
\hline 13 & 4.7828 & 4.6754 & 0.1073 \\
\hline 15 & 1.8760 & 1.7912 & 0.0848 \\
\hline 16 & 3.7387 & 3.5705 & 0.1682 \\
\hline 18 & 3.4788 & 3.2769 & 0.2019 \\
\hline 21 & 3.4877 & 3.2843 & 0.2035 \\
\hline 22 & 4.7962 & 4.4561 & 0.3401 \\
\hline \multirow[t]{2}{*}{23} & 25.3734 & 24.2517 & 1.1217 \\
\hline & 162.5336 & 158.2094 & 4.3242 \\
\hline
\end{tabular}

Only when ten sets of data as shown in Table III are obtained for all samples, learning coefficient can then be generated.

\section{Generator's contribution to a retailer's demand at the receiving end}

For the IEEE 24 bus system with ten generators and ten samples with four samples as base case, there

are

$$
10 \times(10-4)=70 \text { of } \alpha_{1}, \beta_{1} \text { and } \gamma_{1}
$$

respectively, totalling up to 210 coefficients. However, the trends of the coefficients are the subject under study, which for representation purposes are plotted on three graphs illustrated below:

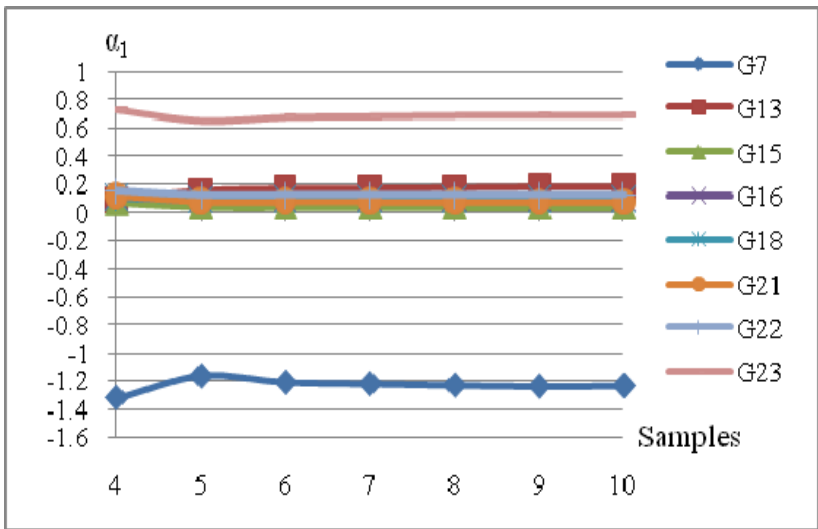

Fig. 4. $\alpha_{1}$ vs. samples 


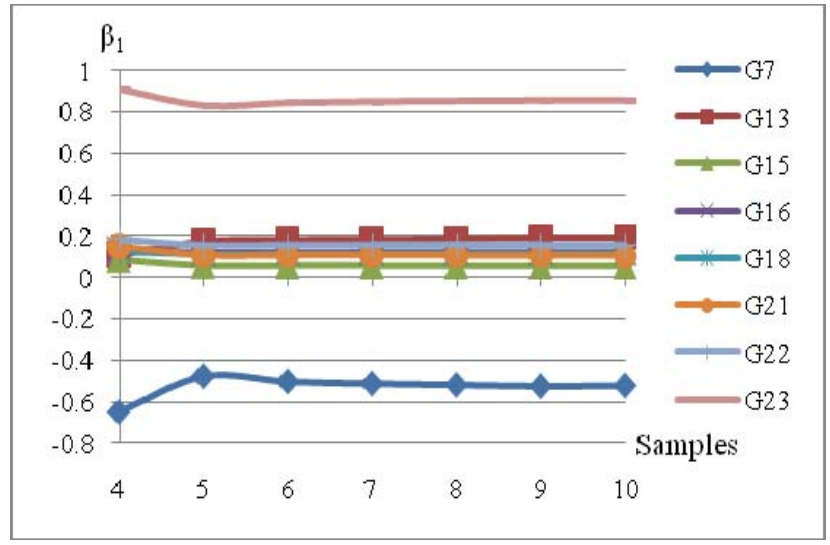

Fig. 5. $\beta_{1}$ vs. samples

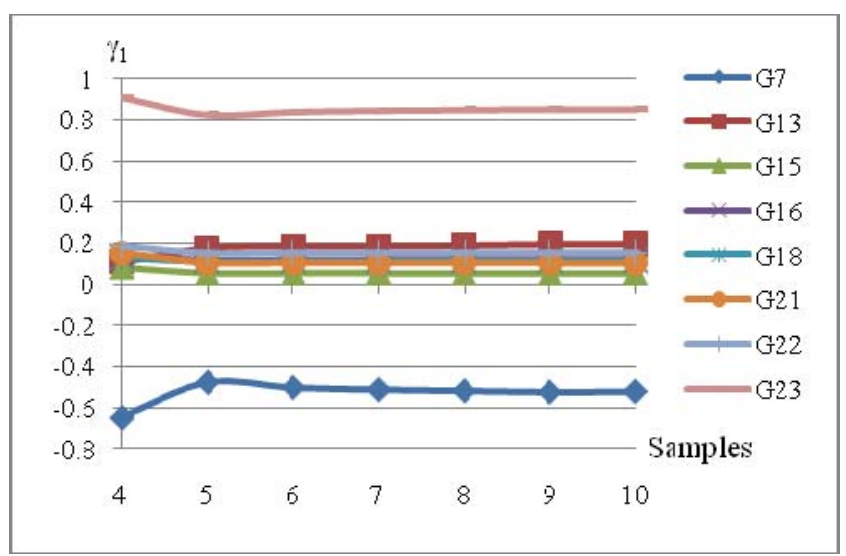

Fig. 6. $Y_{1}$ vs. samples

The number of trends on a single graph (Figure 4, 5, 6 respectively) is equivalent to the number of generators supplying load 8 . This translates to 8 trends in each graph, with each generator having 3

trends: one for $\alpha_{1}, \beta_{1}$ and $\gamma_{1}$ respectively. Notice that

all trends converge with increasing number of

samples. Using the values of $\alpha_{1}, \beta_{1}$ and $\gamma_{1}$ at the

point of ten samples to predict the generator's contribution to a retailer's demand at the receiving end, the results obtained is highly accurate with deviation from the actual results, obtained from load flow by only a mere $0.03 \%$. Hence the better the representation of data/samples are, the more precise the prediction will be.

Another observation from the tracing results and the coefficient trends is such that generators with menial contribution to the demanded power has near zero coefficients, while generators with larger contribution, notably generators 7 and 23 have greater values, either positively or negatively. This is a valuable insight which would prove useful to retailers in the decision of power purchasing from respective generators, which done right would save the retailer from unrecovered cost.

Retailer's demand and the associated loss in a transaction

With the same procedure, the 210 coefficients of $\alpha_{2}, \beta_{2}$ arcl $\gamma_{2}$ are generated and presented in graphical form:

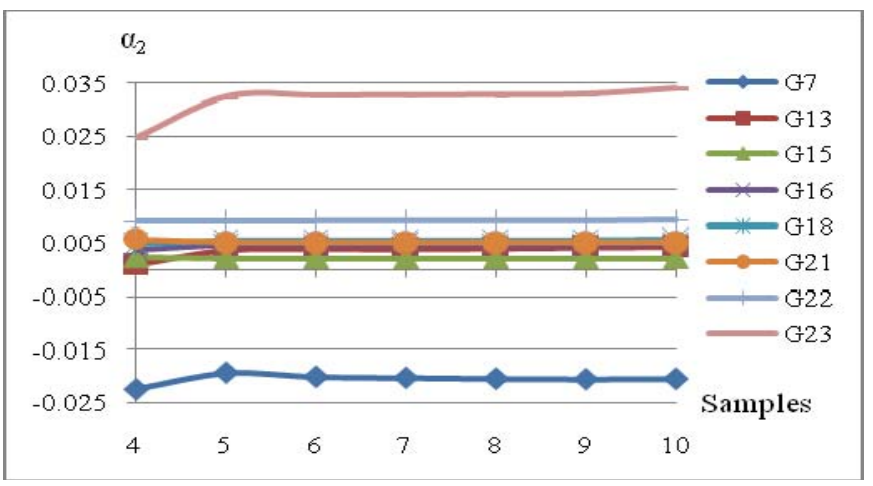

Fig. 7. $\alpha_{2}$ vs. samples

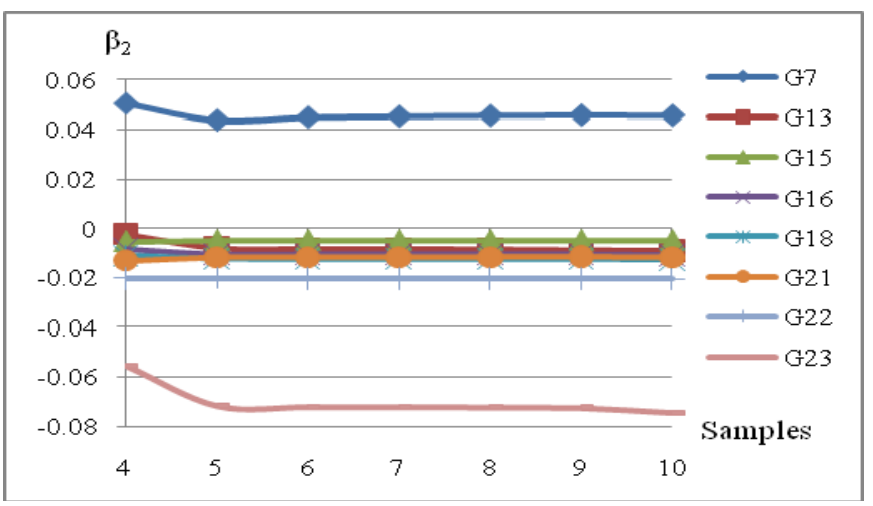

Fig. 8. $\beta_{2}$ vs. samples 


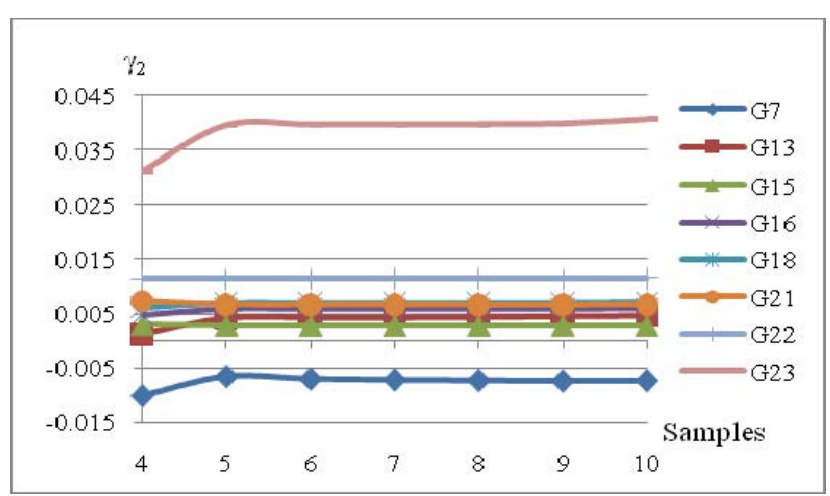

Fig. 9. $Y_{2}$ vs. samples

A prevailing trend can be observed in Figure 7, 8, 9, where the generators with larger contribution notably generators 7 and 23 have greater values, either positively or negatively, while the other generators' coefficients tend to cluster along the zero line. This corroborates with the fact that loss is proportional with power supplied.

There is an interesting revelation from the curve of $\mathrm{G} 23$ (Figure 7, 8, 9). Notice that the trend increases either positively (Figure 7,9 ) or negatively (Figure 8), which indicates an increasing loss associated to G23 supplying load 8 , while the other curves are relatively straight. As $P_{d}$ increases, G23 assumes the prime supplier role, previously held by $\mathrm{G} 7$, which has now reached its maximum allowable operating limits.

This discovery allows the retailer to discern the choice of generator to purchase power from, to supply a marginal increase of demand of a given load, with the function to minimize losses in the transaction, which in turn also minimizes congestion [8], by merely purchasing power from other generators with lower losses.

\section{ConCLUSION}

As observed, data interpretation is made more direct with close relations to the condition of the power system under analysis. This improvisation in many ways improves the convention used in [5] for the Upstream and Downstream Algorithm. A common matrix, which is the bus power level matrix, is created while changes only apply to the flow related matrices (sending and receiving). The decomposed final solution gives revelation to the loss and wheeling factor, an easy way to assess extent of use of the system by either generator or load. Simplification of the method also contributes to the reduction in computation steps and time, since execution can be done with minimal programming.
Converging trend of the learning coefficients underpins the learning coefficient method in performing prediction, where given sufficient samples and spread of samples, prediction done for an oncoming demand could be done to a highly accurate degree. Other valuable information from the trends if harnessed would aid as an effective decision making tool in optimization of profit and operation in a deregulated market, for both generator company and retailer.

\section{REFERENCES}

[1]Perumal.N., Mukerjee.R.N., \& Syafruddin, M. (2010). System Reliability by Prediction of Generator Output and Losses in a Competitive Energy Market. World Academy of Science, Engineering and Technology 2010, Volume 62, Feb 2010., (pp. 665-669). Penang.

[2]Putnam, Hayes \& Bartlett, Inc. (1996). Technical Issues, Methods and Tools in Emerging Energy Market Structures. California: Electric Power Research Institute (EPRI).

[3]Fernando L. Alvarado, R. J. (1997). Open Transmission Access: An Efficient Minimal Role for the ISO. Proc. 30th Hawaii International Conference on System Sciences: Advanced Technology Track - Volume 5. pg 571 .

[4]Bialek, J., Kattuman, P. (2004). Proportional sharing assumption in tracing methodology. Generation, Transmission and Distribution, IEE Proceedings-.

[5]J.Bialek. (1996). Tracing the Flow of Electricity. IEE Proc.-Gener. Trans. Distrib. Vol. 143, No 4, July 1996.

[6]Bergen, A. R., \& Vittal, V. (1999). Power Systems Analysis (2nd Edition ed.). Pearson.

[7]Grigg, Wong, C., Albrecht, P., Allan, P., Bhavaraju, R., Billinton, M., et al. (1996). The IEEE Reliability Test System-1996. A report prepared by the Reliability Test System Task Force of the Application of Probability Methods Subcommittee. IEEE Transactions on Power Systems, Volume 14 (Issue 12), 1010 - 1020.

[8]Pop, D., Kilyeni, S., Andea, P., \& Barbulescu, C. (2009). Congestion risk management using the probable value of congestion and the optimization of the operating regimes Case study for Western part of Romanian Power System. EUROCON 2009, EUROCON '09. 
Am. J. Sci. Ind. Res., 2010, 1(3): 463-471

IEEE . St.-Petersburg

[9]Rosado, S., Abdel-Rahman, K., \& HadjSaid, N. (2001). Tracing the Path of Electric Power Flow - A Study for Deregulated Power Systems. Power Engineering Society Winter Meeting, 2001. IEEE.

[10] Alaywan, Z. (2003). Impact of de-regulation on system operation in California. Power Engineering Society General Meeting, 2003, IEEE

[11] Guoxin, X., Chongqing, K., Gaofeng, Y., \& Zhiwei, W. (2006). A Novel Flexibility Evaluating Approach for Power System Planning under Deregulated Environment. International Conference on Power System Technology, 2006. 